\title{
Reciprocity in parity violating non-Hermitian systems
}

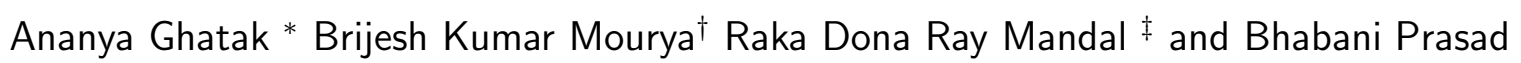 \\ Mandal \$ \\ Department of Physics, Banaras Hindu University, Varanasi-221005, INDIA.
}

\begin{abstract}
Reciprocity is shown so far only when the scattering potential is either real or parity symmetric complex. We extend this result for parity violating complex potential by considering several explicit examples: (i) we show reciprocity for a PT symmetric (hence parity violating) complex potential which admits penetrating state solutions analytically for all possible values of incidence energy and (ii) reciprocity is shown to hold at certain discrete energies for two other parity violating complex potentials.
\end{abstract}

*e-mail address: gananya04@gmail.com

${ }^{\dagger}$ e-mail address: brijeshkumarbhu@gmail.com

${ }^{\ddagger}$ e-mail address: rakad.ray@gmail.com

$\S$ e-mail address: bhabani.mandal@gmail.com, bhabani@bhu.ac.in 


\section{Introduction}

Over a decade and half fully consistent quantum theory for non-Hermitian systems [1]-3] have been developed extensively with its application in different branches of physics [4]31]. Recently scattering due to complex potentials has attracted huge attention due to its rich properties and wide applicabilities and usefulness in the study of different optical systems [4]-[7]. Exceptional points (EPs) [8]-[10], spectral singularity (SS) [11]-[14], invisibility [13]-[16], reciprocity [13]-[17], critical coupling (CC) [18]-22] and coherent perfect absorption (CPA) [23]-37] are among the interesting features of complex scattering. In particular, the CPA which is time reversed counter part of lasing effect has become the center of all such studies in optics due to the discovery of anti-laser [23]-[25] which has a number of technological implications. Other exciting feature is reciprocity which is the topic of discussion in the present work. In the case of scattering due to real potential, transmission coefficient $\left(R_{l}\right)$ for left incident particle is always equal to that $\left(R_{r}\right)$ of for the right incident particle, and the system is called reciprocal. Reciprocity holds good even for complex potentials which respect parity [38, 39]. However reciprocity is known to be violated in the case of complex potentials which are not parity invariant. In fact PT symmetric non-Hermitian systems are always parity violating and hence non-reciprocal. Reciprocity has not been investigated in details for the PT symmetric non-Hermitian systems and to the best of our knowledge not a single example of parity violating PT-symmetric reciprocal system is known till date. Therefore it is worth investigating reciprocity in parity violating non-Hermitian systems. In this work we show that reciprocity can be restored even for the parity violating non-Hermitian systems. We consider three specific examples in support of our claim. In the first example we consider complexified Morse type potential [13] which admits bound, reflecting, penetrating and free state solutions depending on the energy of the incident particle. We show that reciprocity is restored for the entire range of incident energy even though the potential is parity violating and complex, admitting penetrating state solutions. In the case of reflecting states of the same model and in the case of parity violating (PT symmetric or non PT-symmetric) non-Hermitian double delta potential [40], we show that reciprocity is valid only at some specific value of incident energy. These results are shown graphically for the last two models. The most interesting part of the non-Hermitian double delta potential is that the reciprocity is restored only at extreme situations where spectral singularity or reflectionlessness occurs.

The plan of the paper is as follows. We systematically analyse the conditions of reciprocity in the case of both parity symmetric as well as parity violating real potential in section 2 . In section 3 we use the result of section 2 to show the reciprocity for three parity violating complex potentials. Section 4 is kept for summary and discussion. 


\section{Reciprocity : Real Potential}

In this section we obtain the general condition for reciprocity in usual quantum mechanics. The arguments are valid for both real and complex scattering and will be used in the later section. To demonstrate this we consider the general solution of Schroedinger equation for the scattering state as the superposition of the two independent solutions as,

$$
\psi(x)=a U(x)+b V(x)
$$

where $U(x)$ and $V(x)$ are two independent scattering state solutions and $a, b$ are any complex numbers. Now in the case of scattering, $U(x)$ and $V(x)$ are written in general asymptotic form, so that the wave function for $x \rightarrow+\infty$ is written as,

$$
\begin{aligned}
\psi^{+}(x) & =a\left(u_{1}^{+}(k) e^{i k x}+u_{2}^{+}(k) e^{-i k x}\right)+b\left(v_{1}^{+}(k) e^{i k x}+v_{2}^{+}(k) e^{-i k x}\right) \\
& =\left(a u_{1}^{+}(k)+b v_{1}^{+}(k)\right) e^{i k x}+\left(a u_{2}^{+}(k)+b v_{2}^{+}(k)\right) e^{-i k x}
\end{aligned}
$$

Similarly for $x \rightarrow-\infty$ the wave function is written as,

$$
\psi^{-}(x)=\left(a u_{1}^{-}(k)+b v_{1}^{-}(k)\right) e^{i k x}+\left(a u_{2}^{-}(k)+b v_{2}^{-}(k)\right) e^{-i k x}
$$

From the Eqs. (2],3) we can calculate the scattering amplitudes.

For left incidence: $a u_{2}^{+}(k)+b v_{2}^{+}(k)=0$, and hence $r_{l}$ is evaluated as,

$$
r_{l}=\frac{u_{2}^{+} v_{2}^{-}(k)-v_{2}^{+} u_{2}^{-}(k)}{-v_{2}^{+} u_{1}^{-}(k)+u_{2}^{+} v_{1}^{-}(k)}
$$

For right incidence: $a u_{1}^{-}(k)+b v_{1}^{-}(k)=0$, so

$$
r_{r}=\frac{v_{1}^{-} u_{1}^{+}(k)-u_{1}^{-} v_{1}^{+}(k)}{-v_{2}^{+} u_{1}^{-}(k)+u_{2}^{+} v_{1}^{-}(k)}
$$

From Eqs. (4/5) the condition for reciprocity $\left(R_{l}=R_{r}\right)$ is written as,

$$
\begin{aligned}
u_{2}^{+} v_{2}^{-}(k)-v_{2}^{+} u_{2}^{-}(k) & =v_{1}^{-} u_{1}^{+}(k)-u_{1}^{-} v_{1}^{+}(k) \\
\text { or }\left|u_{2}^{+} v_{2}^{-}(k)-v_{2}^{+} u_{2}^{-}(k)\right| & =\left|v_{1}^{-} u_{1}^{+}(k)-u_{1}^{-} v_{1}^{+}(k)\right|
\end{aligned}
$$

Now we discuss parity symmetric and parity violating cases separately using these general results.

\subsection{Parity symmetric potential}

For a parity symmetric potential (real or complex) the general wave functions are written in terms of the odd and even parity solutions. We consider $U(x)=U(-x)$ and $V(x)=$ $-V(-x)$. This implies

$$
U(x \rightarrow \infty) \equiv U^{+}(x) \longrightarrow U^{+}(-x) \equiv U(x \rightarrow-\infty)=U^{-}(x) ;
$$


and

$$
V(x \rightarrow \infty) \equiv V^{+}(x) \longrightarrow V^{+}(-x) \equiv-V(x \rightarrow-\infty)=-V^{-}(x) ;
$$

From the above equation Eq. (8) we see that $U^{+}(-x)=U^{-}(x)$ and hence we obtain,

$$
\begin{array}{ccc}
u_{1}^{+}(k) e^{-i k x}+u_{2}^{+}(k) e^{i k x} & =u_{1}^{-}(k) e^{i k x}+u_{2}^{-}(k) e^{-i k x}, \\
\text { i.e. } & u_{1}^{+}(k)=u_{2}^{-}(k) \quad ; \quad u_{2}^{+}(k)=u_{1}^{-}(k)
\end{array}
$$

Similarly due to $V^{+}(-x)=-V^{-}(x)$ we get,

$$
v_{1}^{+}(k)=-v_{2}^{-}(k) ; v_{2}^{+}(k)=-v_{1}^{-}(k)
$$

Due to these relations in Eq. (9) and Eq. (10), the condition of reciprocity in Eq. (6) is satisfied and the reflection coefficients as well as the amplitudes are equal (i.e. $r_{l}=r_{r}$ and $R_{l}=R_{r}$ ) for arbitrary parity symmetric potential.

\subsection{Parity violating real potential}

For a real potential, the wave function is always chosen as real in the form, $\psi(x)=$ $1 / 2\left\{\phi(x)+\phi^{*}(x)\right\}$ where $\phi^{*}(x)$ and $\phi(x)$ are two independent solution of Schroedinger equation. From the asymptotic forms of $\psi$ in Eq. (21) and (3) we have the following equations for $\psi^{-*}=\psi^{-}$and by considering left incidence case we obtain

$$
\begin{aligned}
& a^{*} u_{1}^{-*}(k)+b^{*} v_{1}^{-*}(k)=a u_{2}^{-}(k)+b v_{2}^{-}(k) \\
\Rightarrow & \frac{-v_{2}^{+}(k)^{*} u_{1}^{-*}(k)+u_{2}^{+}(k)^{*} v_{1}^{-*}(k)}{u_{2}^{+}(k)^{*}}=\frac{-v_{2}^{+} u_{2}^{-}(k)+u_{2}^{+}(k) v_{2}^{-}(k)}{u_{2}^{+}(k)} \\
\Rightarrow & N\left(r_{l}\right)=\frac{u_{2}^{+}(k)}{u_{2}^{+}(k)^{*}}\left[D\left(r_{l}\right)\right]^{*}, \text { thus } r_{l}=\frac{u_{2}^{+}(k)}{u_{2}^{+}(k)^{*}} \frac{\left[D\left(r_{l}\right)\right]^{*}}{\left[D\left(r_{l}\right)\right]}
\end{aligned}
$$

where $N\left(r_{l}\right)$ and $D\left(r_{l}\right)$ denote the numerator and denominator respectively in the expression of $\left(r_{l}\right)$ in equation (4).

$$
N\left(r_{r}\right)=\frac{u_{1}^{-}(k)}{u_{1}^{-}(k)^{*}}\left[D\left(r_{r}\right)\right]^{*}, \text { thus } r_{r}=\frac{u_{1}^{-}(k)}{u_{1}^{-}(k)^{*}} \frac{\left[D\left(r_{r}\right)\right]^{*}}{\left[D\left(r_{r}\right)\right]}
$$

It is clear from equations (11) and (12) that the magnitudes of $r_{l}$ and $r_{r}$ are same, they only differ by a phase. Thus in this parity violating case $R_{l}=R_{r}$ even though $r_{l} \neq r_{r}$. This proves the reciprocity for parity invariant real scattering case.

\section{$3 \quad$ Parity violating complex potential}

In this section we consider three different cases of parity violating complex potential to study the reciprocity. We complexify Morse type potential, which admits bound, 
reflecting, penetrating and free state solutions in two different way. We show that the scattering state for this PT symmetric complex potential is reciprocal only at some specific incident energy, whereas the penetrating states are always reciprocal, even though the potential violate parity symmetry. In another case we consider PT-symmetric as well as non PT-symmetric but Parity violating complex double delta potential to show that reciprocity is restored only at the energy values where SS and reflectionlessness occur.

\subsection{Morse-type potential with scattering states}

The one dimensional real Morse-type potential [13] is written as,

$$
V^{I}(x)=V_{0} \cosh ^{2} \mu^{\prime}\left\{\tanh \left[\left(x-\mu^{\prime} d\right) / d\right]+\tanh \left(\mu^{\prime}\right)\right\}^{2}
$$

This potential admits bound, reflecting, penetrating and free state solutions depending on the energy of the incident particle. We complexified this potential as $\mu^{\prime} \rightarrow i \mu$ so that the corresponding Hamiltonian becomes PT-symmetric non-Hermitian. The Schroedinger equation for this case is written as,

$$
d^{2} \psi_{m} / d z^{2}+\left[\epsilon-v \cosh (2 i \mu)-v \sinh (2 i \mu) \tanh z+v \cosh ^{2}(i \mu) \operatorname{sech}^{2} z\right] \psi_{m}=0
$$

where, $z=(x-i \mu d) / d, v=\left(2 m d^{2} / \hbar^{2}\right) V_{0}, \epsilon=\left(2 m d^{2} / \hbar^{2}\right) E$. Here $z^{P T}=-z$ and $z^{P} \neq \pm z$, so the above potential is invariant only under PT-transformation. The scattering states solutions of Schroedinger equation for this non-Hermitian potential are,

$$
\begin{aligned}
U_{m}(z)= & N e^{\frac{1}{2} i\left(k_{+}-k_{-}\right) z}\left(e^{z}+e^{-z}\right)^{\frac{1}{2} i\left(k_{+}+k_{-}\right) z} \\
\cdot & F\left(-\frac{1}{2} i k_{+}-\frac{1}{2} i k_{-}+\frac{1}{2}-\gamma,-\frac{1}{2} i k_{+}-\frac{1}{2} i k_{-}+\frac{1}{2}+\gamma ;\left|1-i k_{+}\right| ; \frac{e^{-z}}{e^{z}+e^{-z}}\right) \\
V_{m}(z)= & N e^{\frac{1}{2} i\left(k_{+}-k_{-}\right) z}\left(e^{z}+e^{-z}\right)^{\frac{1}{2} i\left(k_{+}+k_{-}\right) z} \cdot\left(\frac{e^{-z}}{e^{z}+e^{-z}}\right) \\
& F\left(\frac{1}{2} i k_{+}-\frac{1}{2} i k_{-}+\frac{1}{2}-\gamma, \frac{1}{2} i k_{+}-\frac{1}{2} i k_{-}+\frac{1}{2}+\gamma ;\left|1+i k_{+}\right| ; \frac{e^{-z}}{e^{z}+e^{-z}}\right) .
\end{aligned}
$$

where

$$
\gamma=\sqrt{-v \cos ^{2} \mu+1 / 4} ; \quad k_{+}=\sqrt{\epsilon-v e^{2 i \mu}} ; \quad k_{-}=\sqrt{\epsilon-v e^{-2 i \mu}}
$$

The general scattering wave function for this Morse-like potential is written in the superposition form of the two independent solutions as,

$$
\psi_{m}(z)=A U_{m}(z)+B V_{m}(z)
$$

with $A$ and $B$ as the arbitrary constants using the standard properties of hyper-geometric functions the asymptotic forms of $\psi_{1}$ and $\psi_{2}$ are written in equivalent notations of Eqs. (21) and (3) as,

$$
\begin{aligned}
& u_{m 1}^{+}=N ; u_{m 2}^{+}=0 ; v_{m 1}^{+}=0 ; v_{m 2}^{+}=N ; \\
& u_{m 1}^{-}=N G 2(k) ; u_{m 2}^{-}=N G 1(k) ; v_{m 1}^{-}=N G 4(k) ; v_{m 2}^{-}=N G 3(k) ;
\end{aligned}
$$




$$
\text { with } \begin{aligned}
G 1 & =\frac{\Gamma\left(1-i k_{+}\right) \Gamma\left(i k_{-}\right)}{\Gamma\left(-\frac{1}{2} i k_{+}+\frac{1}{2} i k_{-}+\frac{1}{2}+\gamma\right) \Gamma\left(-\frac{1}{2} i k_{+}+\frac{1}{2} i k_{-}+\frac{1}{2}-\gamma\right)} \\
G 2 & =\frac{\Gamma\left(1-i k_{+}\right) \Gamma\left(-i k_{-}\right)}{\Gamma\left(-\frac{1}{2} i k_{+}-\frac{1}{2} i k_{-}+\frac{1}{2}-\gamma\right) \Gamma\left(-\frac{1}{2} i k_{+}-\frac{1}{2} i k_{-}+\frac{1}{2}+\gamma\right)} \\
G 3 & =\frac{\Gamma\left(1+i k_{+}\right) \Gamma\left(i k_{-}\right)}{\Gamma\left(\frac{1}{2} i k_{+}+\frac{1}{2} i k_{-}+\frac{1}{2}+\gamma\right) \Gamma\left(\frac{1}{2} i k_{+}+\frac{1}{2} i k_{-}+\frac{1}{2}-\gamma\right)} \\
G 4 & =\frac{\Gamma\left(1+i k_{+}\right) \Gamma\left(-i k_{-}\right)}{\Gamma\left(\frac{1}{2} i k_{+}-\frac{1}{2} i k_{-}+\frac{1}{2}-\gamma\right) \Gamma\left(\frac{1}{2} i k_{+}-\frac{1}{2} i k_{-}+\frac{1}{2}+\gamma\right)}
\end{aligned}
$$

The total wave function behaves asymptotically as,

$$
\begin{aligned}
& \psi_{m}^{+}(z \rightarrow+\infty)=A N e^{i k_{+} z}+B N e^{-i k_{+} z} ; \\
& \psi_{m}^{-}(z \rightarrow-\infty)=N\left[(A G 2+B G 4) e^{i k_{-} z}+(A G 1+B G 3) e^{-i k_{-} z}\right] ;
\end{aligned}
$$

The left and right handed reflection amplitudes which can be constructed by using the Eqs. (4), (5) and (18) (or also can be calculated by (201)) are written as,

$$
r_{l}=\frac{G 1(k)}{G 2(k)} ; r_{r}=-\frac{G 4(k)}{G 2(k)} .
$$

The condition for reciprocity becomes

$$
|G 1(k)|=|G 4(k)|
$$

This can always be seen directly from equation (6).

Eq. (22) is only satisfied for discrete values of ' $k$ ' i.e. only for discrete values of incident particle energy (Fig. 1). Thus for this PT-symmetric non-Hermitian potential one gets reciprocity for certain particle energies without obtaining unitarity. Fig. 1 is showing the discrete incidence energies for which the scattering is reciprocal even for a PT symmetric non-Hermitian system.

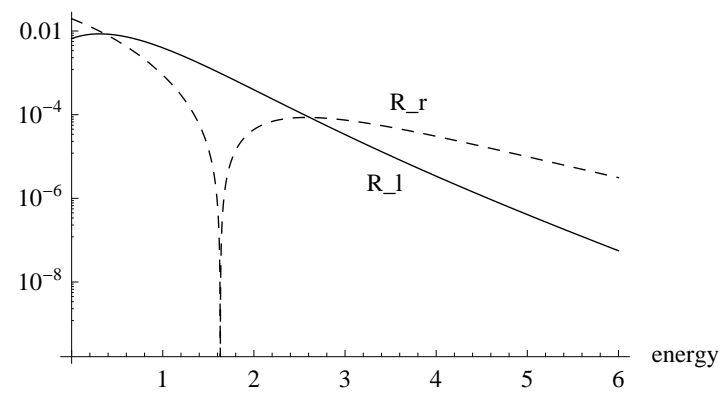

(a)

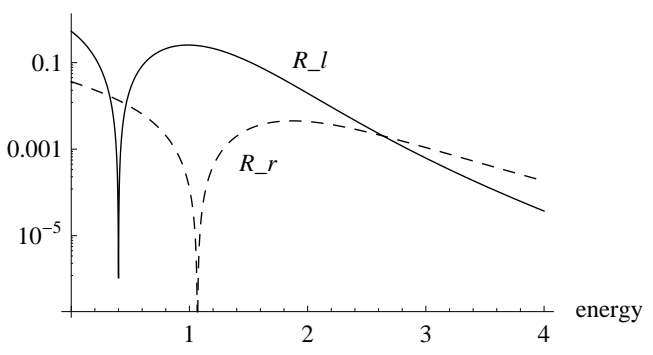

Fig.1: Shows different points where reciprocity is restored even for PT-symmetric nonHermitian system. In 1(a) we have two energy points (in atomic unit) where $R_{l}=R_{r}$ for $v=1.9$ and $\mu=\pi / 5$. 1 (b) shows three such energy points (with $\mu=\pi / 10$ ) where reciprocity is restored. 


\subsection{Parity violating non-Hermitian double-delta potential}

Let us consider the following double-delta potential,

$$
V^{I I}(x)=\lambda\left[\delta\left(x-\frac{a}{2}\right)-\delta\left(x+\frac{a}{2}\right)\right]
$$

where $\lambda$ and $a$ are constant parameters. This potential becomes (i) PT-symmetric nonHermitian for imaginary $\lambda$ and (ii) non PT-symmetric non-Hermitian for complex $\lambda$. In either cases the non-Hermitian potential is parity violating. The reflection amplitudes for left and right incident particle with energy $E$ are written as,

$$
\begin{aligned}
& r_{l}=\frac{\frac{2 i \lambda}{2 k}\left(1-\frac{\lambda}{2 k}\right) \sin (k a)}{\left[1+\left(\frac{\lambda}{2 k}\right)^{2}\left(e^{2 i k a}-1\right)\right]} ; \\
& r_{r}=-\frac{\frac{2 i \lambda}{2 k}\left(1+\frac{\lambda}{2 k}\right) \sin (k a)}{\left[1+\left(\frac{\lambda}{2 k}\right)^{2}\left(e^{2 i k a}-1\right)\right]} ; \text { with } k=\sqrt{E} \text { in atomic unit. }
\end{aligned}
$$

It is easy to see from these equations of reflection amplitudes that scattering due to this non-Hermitian double delta potential is non-reciprocal independent of the fact that PTsymmetry is broken or not. Further we show that reciprocity is achieved at some special situations where spectral singularity or reflectionlessness occurs. From Eq. 24 it is easily seen that at the energies $E_{*}$ for which $\left[\cos \left(2 k_{*} a\right)+i \sin \left(2 k_{*} a\right)\right]+\left(\frac{k_{*}}{\lambda}\right)^{2}=1$ both $r_{l}$ and $r_{r}$ are infinite. This is nothing but the condition of spectral singularity for the energy of the particle incident from either side. On the other situation reciprocity is restored due to the case of bidirectional reflectionlessness $\left(r_{l}=r_{r}=0\right)$ at discrete incidence energies $E_{* *}=\frac{n^{2} \pi^{2}}{2 a^{2} m}$. Fig. 2 explains both the conditions where the left and right handed reflection amplitudes behave in exactly similar way.
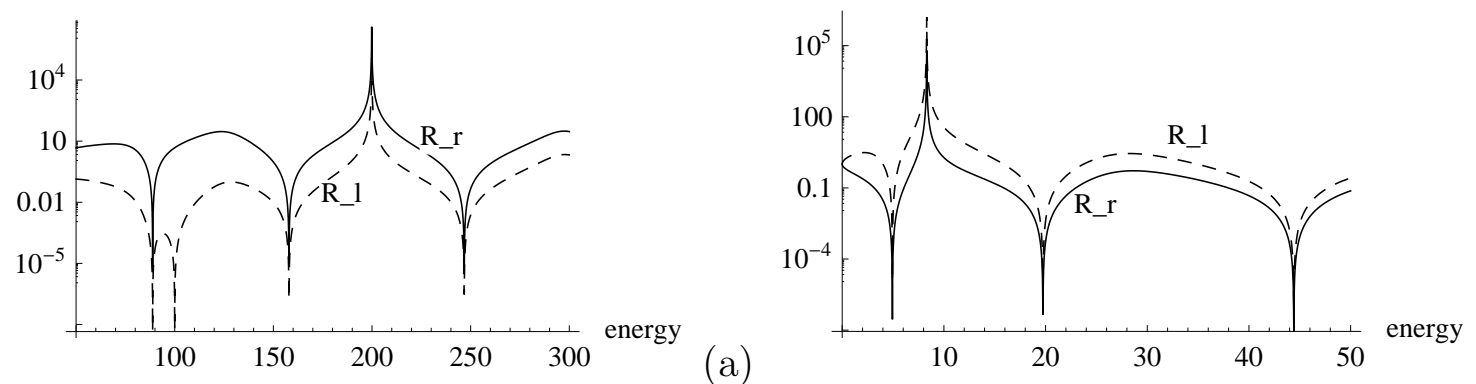

Fig. 2: ( $a, b)$ shows the restoration of reciprocity for parity asymmetric double delta non-Hermitian potential when $a=1, \lambda=20 i$ and $a=-1, \lambda=2.01 i-6.1$ respectively at different discrete energies. Reciprocity and SS or reflectionlessness occurs at the same energy values. 


\subsection{Penetrating state of a complex potential and reciprocity}

The potential in Eq. [13] is complexified in PT-symmetric manner by taking the parameter $d$ imaginary (i.e. $d \rightarrow i d$ ) and keeping the other parameters real. For this case the time independent Schroedinger equation (TISE) takes the form,

$$
\frac{\hbar^{2}}{2 m d^{2}} \frac{d^{2} \psi}{d z^{2}}+V^{I}(z) \psi=E \psi
$$

where $\mathrm{z}$ is taken as $z=-i X / d-\mu$, with $X=x+i \zeta$. Note that the differential term in this equation comes with wrong sign due to the presence of $d^{2}$ term. However this equation can be interpreted as TISE for a upside down potential of the original one with energy eigenvalues $(-\mathrm{E})$. It behaves like a potential barrier with a maxima at $\mathrm{x}=0$. We have penetrating state solutions if the particle has negative energy with magnitude less than $v e^{-2 \mu}$. On the other hand it accepts free states when the energy of the particle is more than the barrier height.

The penetrating state solutions are given by,

$$
\begin{aligned}
\psi_{1}(z)= & N e^{-a z}\left(e^{z}+e^{-z}\right)^{-b z} F\left(b+\frac{1}{2}-\sqrt{-v \cosh ^{2}(\mu)+1 / 4},\right. \\
& \left.b+\frac{1}{2}+\sqrt{-v \cosh ^{2}(\mu)+1 / 4} ;|a+b+1| ; \frac{e^{-z}}{e^{z}+e^{-z}}\right) \\
\psi_{2}(z)= & N e^{b z}\left(e^{z}+e^{-z}\right)^{a z} F\left(-a+\frac{1}{2}-\sqrt{-v \cosh ^{2}(\mu)+1 / 4}\right. \\
& \left.-a+\frac{1}{2}+\sqrt{-v \cosh ^{2}(\mu)+1 / 4} ;|1-a-b| ; \frac{e^{-z}}{e^{z}+e^{-z}}\right)
\end{aligned}
$$

with

$$
a^{2}+b^{2}=-\epsilon-v \cosh 2 \mu ; \quad 2 a b=-v \sinh 2 \mu ;
$$

and $k_{+}=\sqrt{\epsilon+v e^{2 \mu}}, k_{-}=\sqrt{\epsilon+v e^{-2 \mu}}$ so that

$$
a=-1 / 2\left(i k_{+}+i k_{-}\right) ; \quad b=1 / 2\left(-i k_{+}+i k_{-}\right) .
$$

The wave function for this penetrating state is written in a general form as,

$$
\psi(z)=A \psi_{1}(z)+B \psi_{2}(z)
$$

We calculate the left and right handed penetrating amplitudes from the asymptotic behavior of Eq. (29) as,

$$
r_{l}=\frac{P 1}{P 2} \quad r_{r}=-\frac{P 4}{P 2},
$$


where P1, P2, P3, P4 are expressed in terms of Gamma function written as,

$$
\begin{aligned}
P 1 & =\frac{\Gamma\left(1-i k_{+}\right) \Gamma\left(-i k_{-}\right)}{\Gamma\left(-\frac{1}{2} i k_{+}-\frac{1}{2} i k_{-}+\frac{1}{2}+\gamma^{\prime}\right) \Gamma\left(-\frac{1}{2} i k_{+}-\frac{1}{2} i k_{-}+\frac{1}{2}-\gamma^{\prime}\right)} \\
P 2 & =\frac{\Gamma\left(1-i k_{+}\right) \Gamma\left(i k_{-}\right)}{\Gamma\left(-\frac{1}{2} i k_{+}+\frac{1}{2} i k_{-}+\frac{1}{2}-\gamma^{\prime}\right) \Gamma\left(-\frac{1}{2} i k_{+}+\frac{1}{2} i k_{-}+\frac{1}{2}+\gamma^{\prime}\right)} \\
P 3 & =\frac{\Gamma\left(1+i k_{+}\right) \Gamma\left(-i k_{-}\right)}{\Gamma\left(\frac{1}{2} i k_{+}-\frac{1}{2} i k_{-}+\frac{1}{2}+\gamma^{\prime}\right) \Gamma\left(\frac{1}{2} i k_{+}-\frac{1}{2} i k_{-}+\frac{1}{2}-\gamma^{\prime}\right)} \\
P 4 & =\frac{\Gamma\left(1+i k_{+}\right) \Gamma\left(i k_{-}\right)}{\Gamma\left(\frac{1}{2} i k_{+}+\frac{1}{2} i k_{-}+\frac{1}{2}-\gamma^{\prime}\right) \Gamma\left(\frac{1}{2} i k_{+}+\frac{1}{2} i k_{-}+\frac{1}{2}+\gamma^{\prime}\right)}
\end{aligned}
$$

where $\gamma^{\prime}=\sqrt{-v \cosh ^{2}(\mu)+1 / 4}$. Here we note $r_{l} \neq r_{r}$ but $P 1^{*}=P 4 ; \quad P 2^{*}=P 3$ (due to real $k_{+}$and $\left.k_{-}\right)$. Thus the second condition of Eq. (6) is being satisfied so that

$$
R_{l} \equiv\left|r_{l}\right|^{2}=\left|r_{r}\right|^{2} \equiv R_{r}
$$

This implies that we have reciprocity for this non-Hermitian PT-symmetric system for all values of energy. However as expected unitarity is violated, i.e $R+T \neq 1$ (both for left and right handed cases) for this model. We further observe no SS is present for penetrating states and the potential never becomes reflectionless.

\section{Conclusion}

Scattering due to real potential is always reciprocal. Further it has been shown that even complex scattering obeys reciprocity if the potential is parity symmetric. This implies that scattering due to PT symmetric non-Hermitian systems are supposed to be non-reciprocal as PT symmetric non-Hermitian system is essentially parity violating. In this present work we show that reciprocity is valid even for certain PT symmetric nonHermitian systems. In support of our claim we consider, three explicit examples. The scattering states of PT-symmetric complex Morse potential is reciprocal only at certain specific incident energies. In another example we consider PT-symmetric as well as non PT-symmetric complex double delta potential to show restoration of reciprocity only at SS and reflectionless points. Most interesting result is for penetrating states of PT symmetric non-Hermitian Morse potential which is shown to be reciprocal at all incident energy. Unitarity of scattering S-matrix and reciprocity are two important characteristics of scattering theory. Their validity/non validity in the case of complex scattering is extremely important. Our results are one step forward in the investigation of reciprocity of complex scattering. No example of PT-symmetric non-Hermitian potentials is known so far when both unitarity and reciprocity hold good. It will be worth finding more and more parity violating non-Hermitian systems which are reciprocal as well as unitary. 
Acknowledgments BKM and BPM acknowledge the financial support from the Department of Science and Technology (DST), Gov. of India under SERC project sanction grant No. SR/S2/HEP-0009/2012. AG acknowledges the Council of Scientific \& Industrial Research (CSIR), India for Senior Research Fellowship.

\section{References}

[1] C. M. Bender and S. Boettcher, Phys. Rev. Lett. 80, 5243 (1998).

[2] A. Mostafazadeh, Int. J. Geom. Meth. Mod. Phys. 7, 1191(2010) and references therein.

[3] C.M. Bender, Rep. Progr. Phys. 70, 947 (2007) and references therein.

[4] Z. H. Musslimani, K. G. Makris, R. El-Ganainy, and D. N. Christodoulides, Phys. Rev. Lett. 100, 030402 (2008).

[5] C. E. Ruter, K. G. Makris, R. El-Ganainy, D. N. Christodoulides, M. Segev, D. Kip, Nature Phys. 6 192, (2010);

[6] R. El-Ganainy, K. G. Makris, D. N. Christodoulides and Z. H. Musslimani, Opt. Lett. 32, 2632 (2007).

[7] A. Guo et al, Phys. Rev. Lett. 103, 093902 (2009).

[8] T. Kato, Perturbation Theroy of Linear Operators, Springer, Berlin, (1966).

[9] M. V. Berry, Czech. J. Phys. 54, 1039 (2004).

[10] W. D. Heiss, Phys. Rep. 242, 443 (1994).

[11] A. Mostafazadeh, Phys. Rev. Lett. 102, 220402 (2009).

[12] A. Mostafazadeh, M. Sarisaman, Phys. Lett. A 375, 3387 (2011).

[13] A. Ghatak, R. D. Ray Mandal, B. P. Mandal, Ann. of Phys. 336, 540 (2013).

[14] A. Ghatak, J. A. Nathan, B. P. Mandal, and Z. Ahmed, J. Phys. A: Math. Theor. 45, 465305 (2012).

[15] S. Longhi, J. Phys. A: Math. Theor. 44, 485302 (2011).

[16] A. Mostafazadeh, Phys. Rev. A 87, 012103 (2013).

[17] L. Deak, T. Fulop, Ann. of Phys. 327, 1050 (2012).

[18] M. Cai, O. Painter, and K. J. Vahala, Phys. Rev. Lett. 85, 74 (2000). 
[19] J. R. Tischler, M. S. Bradley, and V. Bulovic, Opt. Lett. 31, 2045 (2006)

[20] S. Dutta Gupta, Opt. Lett. 32, 1483 (2007).

[21] S. Balci, C. Kocabas, and A. Aydinli, Opt. Lett. 36, 2770 (2011).

[22] S. Balci, Er. Karademir, C. Kocabas, and A. Aydinli, Opt. Lett. 36, 3041 (2011).

[23] C. F. Gmachl, Nature 467, 37 (2010).

[24] S. Longhi, Physics 3, 61(2010).

[25] W. Wan, Y. Chong, L. Ge, H. Noh, A. D. Stone, H. Cao, Science 331, 889 (2011).

[26] M. Hasan, A. Ghatak, B. P. Mandal, Ann. of Phys. 344, 17 (2014).

[27] A. Ghatak and B. P. Mandal, J. Phys. A: Math. Theor. 45, 355301 (2012).

[28] B. P. Mandal and S S. Mahajan arXiv:1312.0757, (2013).

[29] B. P. Mandal, B. K. Mourya, and R. K. Yadav (BHU), Phys. Lett. A 377, 1043 (2013).

[30] B. P. Mandal, Mod. Phys. Lett. A 20, 655(2005).

[31] B. P. Mandal and A. Ghatak, J. Phys. A: Math. Theor. 45, 444022 (2012) .

[32] N. Liu, M. Mesch, T. Weiss, M. Hentschel, and H. Giessen, Nano Lett. 10, 2342 (2010).

[33] H. Noh, Y. Chong, A. Douglas Stone, and Hui Cao, Phys. Rev. Lett. 108, 6805 (2011).

[34] A. Mostafazadeh and M. Sarisaman, Proc. R. Soc. A 468, 3224 (2012).

[35] S. Longhi, Phys. Rev. A 83, 055804 (2011).

[36] S. Dutta-Gupta, R. Deshmukh, A. Venu Gopal, O. J. F. Martin, and S. Dutta Gupta, Opt. Lett. 37, 4452 (2012).

[37] N. Liu, M. Mesch, T. Weiss, M. Hentschel, and H. Giessen, Nano Lett. 102342 (2010).

[38] Z. Ahmed, Phys. Lett. A 377, 957 (2013).

[39] A Mostafazadeh, arXiv:1405.4212, (2014).

[40] Ram Narayan Deb, Avinash Khare, Binayak Dutta Roy, Phys. Lett. A 307215 (2003). 